\title{
Efeito do treinamento de força na flexibilidade: uma revisãó sistemática
}

\section{Effect of strength training on flexibility: a systematic review}

\author{
Marilia A. Correia' \\ Annelise L. Menêses ${ }^{1}$ \\ Aluísio H. R. A. Lima' \\ Bruno R. Cavalcante \\ Raphael M. Ritti-Dias
}

\section{RESUMO}

O objetivo do presente estudo foi determinar o efeito do treinamento de força na flexibilidade de indivíduos adultos jovens e idosos. Foi realizada uma revisão sistemática de artigos científicos originais indexados nas bases de dados eletrônicas MEDLINE®, LILACS®, SCIELO®, Web of Knowledge® SCOPUS ${ }^{\mathrm{TM}}$, até o ano de 2012. Foram selecionados estudos que incluíram termos no título e descritores relacionados com a flexibilidade e o treinamento de força. Dezesseis artigos foram incluídos na revisão. Dos cinco estudos com a população idosa, todos reportaram aumento da flexibilidade após o treinamento de força, independente do protocolo e do método/instrumento utilizado. Em indivíduos adultos jovens, sete estudos observaram aumento na flexibilidade com o treinamento de força em pelo menos uma articulação, ao passo que quatro estudos não observaram qualquer alteração. Não foi possível identificar características na amostra ou nos protocolos de treinamento que pudessem diferenciar os estudos que observaram efeitos significantes e não significantes do treinamento de força na flexibilidade. Entretanto, todos os estudos que utilizaram o goniômetro ou o flexímetro reportaram aumento da flexibilidade em pelo menos uma articulação. Em conclusão, o treinamento de força aumenta a flexibilidade de indivíduos idosos, ao passo que em adultos jovens, aumentos da flexibilidade com o treinamento de força são controversos.

\section{PALAVRAS-CHAVE}

Treinamento de Resistência. Amplitude de Movimento Articular. Exercícios de Alongamento Muscular.

\begin{abstract}
The aim of this study was to identify factors that influence the effects of strength training on young adults and elderly flexibility. Systematic review of original scientific articles indexed in the electronic databases MEDLINE®, LILACS®, SCIELO®, Web of Knowledge ${ }^{\circledR}$ SCOPUS ${ }^{\mathrm{TM}}$, by the year 2012, was performed. There were selected studies that included terms in the title and keywords related to flexibility and strength training. Sixteen articles were included in the review. Of the five studies with the elderly population, all reported increased flexibility after strength training, regardless of the protocol and the method / instrument used. In young adults, seven studies observed increases in flexibility following strength training in at least one articulation, while four studies have not found any alteration. It was not possible to identify characteristics in the sample or in the training protocols that could differentiate the studies that observed significant and no significant effects of training on flexibility. However, all studies using goniometer or fleximeter reported increased flexibility in at least one articulation. In conclusion, strength training increases flexibility of elderly individuals, whereas in young adults, increases in flexibility due to strength training are controversial.
\end{abstract}

\section{KEYWORDS}

Resistance Training. Range of Motion, Articular. Muscle Stretching Exercises.
Rev Bras Ativ Fis Saúde p. 3-11 DOI

http://dx.doi.org/10.12820/rbafs.v.19n1p3

1 Grupo de Estudo e Pesquisa em Hemodinâmica, Metabolismo e Exercício

- GEPHEME. Escola Superior de Educação

Física da Universidade de Pernambuco (ESEF/ UPE). 


\section{INTRODUCCÃO}

O treinamento de força tem se tornado popular, visto que promove importantes benefícios para a aptidão musculoesquelética, tais como o aumento da massa muscular ${ }^{5,6}$, dos níveis de força ${ }^{7}$ e da densidade mineral óssea ${ }^{5}$. Essas adaptações melhoram a capacidade funcional e a qualidade de vida dos praticantes $^{8,9} \mathrm{e}$, por isso, o treinamento de força tem sido recomendado para diferentes grupos populacionais ${ }^{5,7}$.

A flexibilidade é um importante componente da aptidão física que está relacionada com as atividades da vida diária ${ }^{10-13}$. Consequentemente, intervenções que aumentem o desempenho nesse componente são importantes para a saúde. Embora estudos venham sugerindo que programas de treinamento de força podem aumentar a flexibilidade ${ }^{1,2,6,14,15}$, esse não é um achado universal, visto que outros estudos não evidenciaram quaisquer alterações na flexibilidade após programas de treinamento de força ${ }^{16-18}$. Essa controvérsia pode ser decorrente dos diferentes procedimentos metodológicos empregados entre os estudos, bem como pelas características dos indivíduos estudados, que diferem amplamente com relação à faixa-etária.

De fato, a faixa etária parece influenciar os níveis de flexibilidade dos indivíduos ${ }^{11}$, uma vez que o processo de envelhecimento pode afetar a rigidez dos tendões implicando negativamente no grau de mobilidade das articulações ${ }^{19}$, ${ }^{20}$. Como o treinamento de força parece aumentar a flexibilidade devido à redução na tensão passiva e na rigidez do músculo esquelético ${ }^{21}$, é possível que os efeitos do treinamento de força sejam mais evidentes nos idosos, que sabidamente apresentam mobilidade prejudicada. Assim, o objetivo do presente estudo foi determinar o efeito do treinamento de força na flexibilidade de indivíduos adultos jovens e idosos.

\section{MÉTODOS}

Trata-se de uma revisão sistemática de artigos originais publicados em periódicos indexados nas bases de dados eletrônicas MEDLINE ${ }^{\circledR}$ (http://www. pubmed.com), LILACS $^{\circledR}$ (http://www.bireme.br), SCIELO ${ }^{\circledR}$ (http://www. scielo.org), Web of Knowledge ${ }^{\circledR}$ (http://www.isiknowledge.com) e SCOPUS $^{\text {TM }}$ (http://www.scopus.com) sobre o efeito do treinamento de força na flexibilidade, publicados até 2012. Não foram incluídos artigos de revisão, artigos sobre o efeito de outras formas de exercício considerados exercícios de força (pilates, pliometria, yoga), artigos que investigaram o efeito de intervenções que incluíram outras modalidades de exercício combinado com o treinamento de força (treinamento de força + treinamento aeróbio ou treinamento de flexibilidade), estudos de caso e estudos sem grupo controle.

A revisão sistemática consistiu em três etapas: busca eletrônica nas bases de dados (etapa 1), seleção e identificação dos artigos elegíveis (etapa 2) e extração dos dados dos estudos incluídos na revisão (etapa 3). Dois pesquisadores realizaram de maneira independente cada etapa do processo de revisão. Os resultados de cada etapa foram comparados por um terceiro avaliador a fim de verificar a concordância dos achados. $\mathrm{Na}$ eventualidade de divergências, o terceiro pesquisador fez a análise final. 


\section{Etapa 1: busca eletrônica nas bases de dados}

Os descritores e os termos utilizados para a busca eletrônica nas bases de dados foram identificados mediante consulta ao MeSH - Medical Subject Headings, através do portal da U.S. National Library of Medicine (NLM) e ao DeCS - Descritores em Ciências da Saúde, através do portal da Biblioteca Virtual em Saúde (BVS).

Os descritores (Range of Motion, Articular; Muscle Stretching Exercises; Exercise; Resistance Training; Weight Lifting; Physical Education and Training e os seus respectivos homólogos na língua portuguesa) e os termos presentes no título (Flexibility; Resistance Training; Resistance Exercise; Strength Training; Weight Training e os seus respectivos homólogos na língua portuguesa) utilizados na busca foram, quando possível, combinados com a aplicação de limites de acordo com as ferramentas de busca avançada disponíveis em cada base de dados. Nessa etapa, 2400 artigos foram identificados na base de dados MEDLINE $^{\circledR}, 34$ na LILACS $^{\circledR}, 15$ na SCIELO $^{\circledR}, 176$ na Web of Knowledge $^{\circledR}$ e 1811 na SCOPUS ${ }^{\mathrm{TM}}$ (Figura 1 ).

\section{Etapa 2: seleção e identificação dos artigos elegíveis}

Para a identificação dos artigos elegíveis para inclusão na revisão, foi realizada uma triagem inicialmente pelo título e, em seguida, pelo resumo. Nessa etapa, 28 artigos foram considerados elegíveis para a inclusão no estudo. Após isso, todos os artigos foram lidos na íntegra e as referências bibliográficas dos artigos incluídos na revisão foram avaliadas, com o objetivo de verificar a existência de artigos elegíveis não identificados nas buscas nas bases de dados. Após exclusão das publicações repetidas, 16 artigos ${ }^{5-12,17-24}$ foram incluídos no estudo (Figura 1).

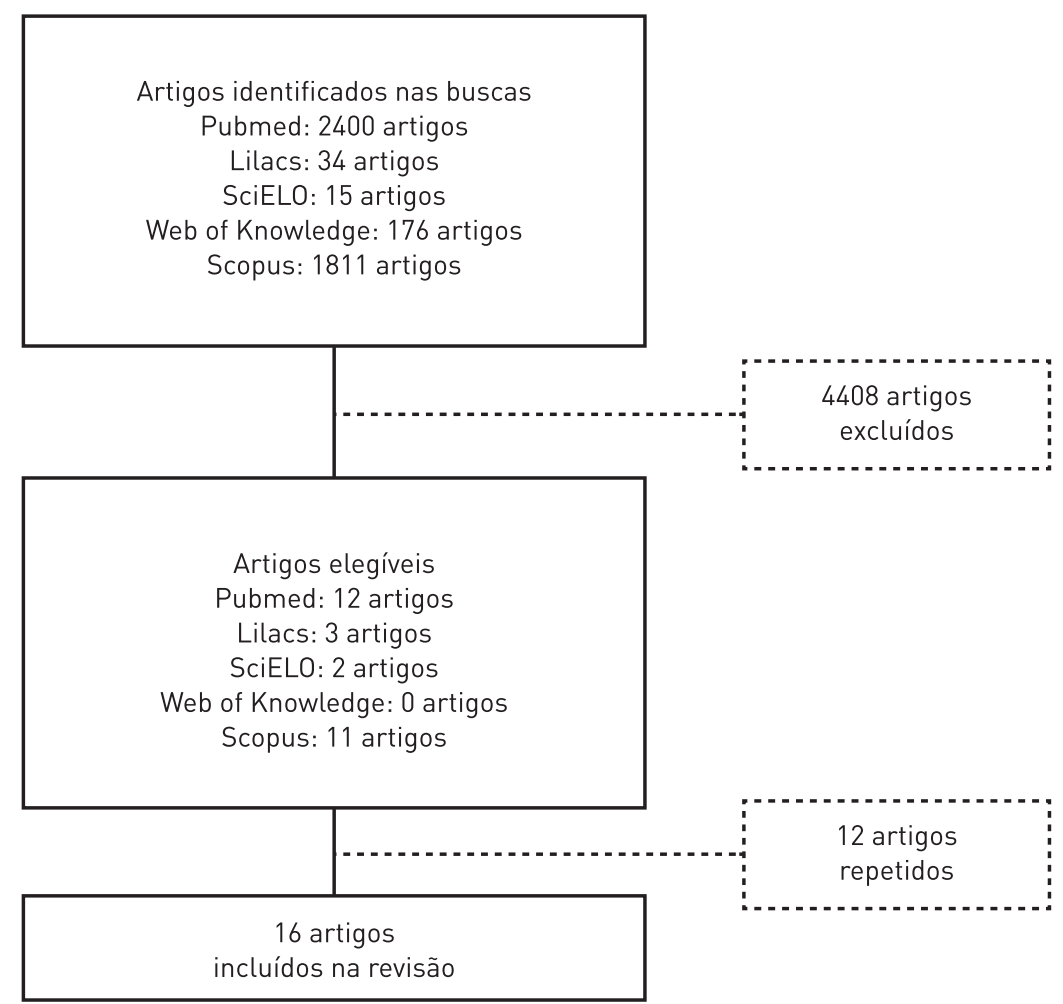


FIGURA 1 - Fluxograma da seleção dos estudos incluídos na revisão.

\section{Etapa 3: extração dos dados}

Em posse dos artigos foi feita a extração dos seguintes dados: (a) referência da publicação, (b) distribuição da amostra em diferentes grupos de intervenção, (c) tamanho da amostra, (d) gênero, (e) idade, (f) características do protocolo de treinamento de força (período de treinamento, frequência semanal, exercícios utilizados, musculatura envolvida, número de séries e intensidade), (g) método ou instrumento utilizados para a avaliação da flexibilidade, (h) comportamento da força após o treinamento e (i) resultados. A extração das informações dos estudos foi feita elaborando-se duas tabelas, de acordo com a faixa-etária da amostra (adultos jovens e idosos).

\section{RESULTADOS}

A Tabela 1 apresenta as características da amostra, os procedimentos metodológicos e os principais resultados dos estudos realizados com adultos jovens. A amostra dos estudos variou de 16 a 132 indivíduos, de ambos os sexos e com idade entre 21 e 51 anos. O período de treinamento variou de quatro a 24 semanas, sendo realizadas entre uma e quatro sessões semanais. A maioria dos estudos incluiu exercícios para os membros superiores e inferiores, no entanto, três estudos ${ }^{1,3,17}$ incluíram apenas exercícios para os membros inferiores. Diferentes instrumentos foram utilizados para a avaliação da flexibilidade, sendo o goniômetro o mais utilizado.

Com relação aos efeitos do treinamento de força com adultos jovens, dos onze estudos no incluídos, sete demonstraram aumento significante na flexibilidade ${ }^{1-4,9,22,23}$, ao passo que destes, seis informaram um aumento da força muscu$\operatorname{lar}^{1-4,9,23}$. A maioria dos estudos que observaram efeitos significantes assim como os que observaram efeito não significante realizaram programas de treinamento de força de 8 a 10 semanas, com três sessões semanais e contemplaram exercícios para os membros superiores, inferiores e tronco, com intensidade variando desde o próprio peso corporal até $100 \%$ da carga máxima suportada pelo indivíduo.

Em adultos jovens, todos os estudos que utilizaram o goniômetro ${ }^{1-4}$ ou o flexímetro ${ }^{20,22}$ reportaram aumento da flexibilidade em pelo menos uma articulação. Entretanto, dos três estudos que utilizaram o teste de sentar e alcançar $^{16,18}$ para avaliação da flexibilidade, dois ${ }^{16,18}$ apresentaram manutenção e $\mathrm{um}^{9}$ apresentou aumento da flexibilidade.

A Tabela 2 apresenta as características da amostra, os procedimentos metodológicos e os principais resultados dos estudos sobre o efeito do treinamento de força na flexibilidade em idosos. A amostra variou de 19 a 48 indivíduos de ambos os sexos. Todos os indivíduos tinham idade entre 65 e 70 anos. Os instrumentos mais utilizados para avaliação da flexibilidade foram o goniômetro e o teste de sentar e alcançar. Todos os estudos reportaram aumento na flexibilidade após o treinamento de força. Destes, três informaram que houve um aumento na força muscular ${ }^{14,15,24}$. Nesses estudos, o período de treinamento variou de quatro a 16 semanas, sendo realizada uma a três sessões semanais, incluindo exercícios para membros superiores, inferiores e tronco, com intensidade variando desde o peso do próprio corpo até $85 \%$ da carga máxima 


\section{suportada pelo indivíduo.}

TABELA 1 - Características da amostra, procedimentos metodológicos e principais resultados dos estudos sobre o efeito do treinamento de força na flexibilidade de adultos jovens.

\begin{tabular}{|c|c|c|c|c|c|c|}
\hline Autor(es) & Grupos & Amostra & Protocolo de treinamento & $\begin{array}{c}\text { Método/ } \\
\text { Instrumento }\end{array}$ & $\begin{array}{l}\text { Comportamento } \\
\text { da força muscular }\end{array}$ & $\begin{array}{l}\text { Efeito após o } \\
\text { treinamento }\end{array}$ \\
\hline $\begin{array}{l}\text { Nóbrega } \\
\text { et al. }{ }^{29}\end{array}$ & $\begin{array}{l}\text { Controle } \\
\text { TF }\end{array}$ & $\begin{array}{l}\mathrm{n}=43(\mathrm{H} / \mathrm{M}) \\
21 \text { anos }\end{array}$ & $\begin{array}{l}12 \text { semanas, } 2 x / \text { semana; } 9 \text { exercícios } \\
\text { (MMSS, MMII e Tronco); } 3 \text { séries de } 8 \text { - } \\
12 \text { reps a } 60 \% \text { de } 1 \text { RM }\end{array}$ & Flexiteste & $\uparrow$ & $\begin{array}{l}\leftrightarrow \text { MMSS } \\
\leftrightarrow \text { MMII } \\
\leftrightarrow \text { Tronco }\end{array}$ \\
\hline $\begin{array}{l}\text { Kim } \\
\text { et al. }^{18}\end{array}$ & $\begin{array}{l}\text { Controle } \\
\text { TF (tradicional) } \\
\text { TF (super lento) }\end{array}$ & $\begin{array}{l}n=35(M) \\
21 \text { anos }\end{array}$ & $\begin{array}{l}\text { TF tradicional } \\
4 \text { semanas, } 3 x / \text { semana; } 5 \text { exercícios } \\
\text { (MMSS e MMII); } 3 \text { séries de } 8 \text { reps a } \\
80 \% \text { de } 1 \text { RM } \\
\text { TF super lento } \\
4 \text { semanas, } 2 x / \text { semana; } 5 \text { exercícios } \\
\text { (MMSS e MMII); } 1 \text { série até a fadiga } \\
\text { muscular momentânea }\end{array}$ & $\begin{array}{l}\text { Teste de } \\
\text { sentar e } \\
\text { alcançar }\end{array}$ & $\uparrow$ & $\leftrightarrow$ MMII e tronco \\
\hline $\begin{array}{l}\text { Mahieu } \\
\text { et al. }{ }^{1}\end{array}$ & $\begin{array}{l}\text { Controle } \\
\text { TF (excêntrico) }\end{array}$ & $\begin{array}{l}\mathrm{n}=64(\mathrm{H} / \mathrm{M}) \\
22 \text { anos }\end{array}$ & $\begin{array}{l}6 \text { semanas, } 1 \text { x/semana; flexão plantar } \\
\text { (MMII); } 3 \text { séries de } 15 \text { reps com o peso } \\
\text { corporal }\end{array}$ & Goniômetro & $\uparrow$ & $\uparrow M M I I$ \\
\hline $\begin{array}{l}\text { Aquino } \\
\text { et al. }{ }^{17}\end{array}$ & $\begin{array}{l}\text { Controle } \\
\text { TF }\end{array}$ & $\begin{array}{l}\mathrm{n}=45(\mathrm{H} / \mathrm{M}) \\
22 \text { anos }\end{array}$ & $\begin{array}{l}8 \text { semanas, } 3 x / \text { semana; flexão de } \\
\text { pernas (MMII); } 3 \text { séries de } 12 \text { reps a } \\
60 \% \text { de } 1 \text { RM }\end{array}$ & $\begin{array}{l}\text { Dinamômetro } \\
\text { isocinético }\end{array}$ & $\uparrow$ & $\leftrightarrow \mathrm{MMII}$ \\
\hline $\begin{array}{l}\text { Morton } \\
\text { et al. }{ }^{2}\end{array}$ & $\begin{array}{l}\text { Controle } \\
\text { TF }\end{array}$ & $\begin{array}{l}\mathrm{n}=36(\mathrm{H} / \mathrm{M}) \\
22 \text { anos }\end{array}$ & $\begin{array}{l}5 \text { semanas, } 3 x / \text { semana; } 20 \text { exercícios } \\
\text { (MMSS e MMII); } 3 \text { séries até a fadiga }\end{array}$ & $\begin{array}{l}\text { Goniômetro } \\
\text { e teste de } \\
\text { elevação de } \\
\text { braço }\end{array}$ & $\uparrow$ & $\begin{array}{l}\stackrel{\leftrightarrow}{M M S S} \\
\uparrow M M I I\end{array}$ \\
\hline $\begin{array}{l}\text { Cyrino } \\
\text { et al. }^{22}\end{array}$ & $\begin{array}{l}\text { Controle } \\
\text { TF }\end{array}$ & $\begin{array}{l}n=16(H / M) \\
23 \text { anos }\end{array}$ & $\begin{array}{l}10 \text { semanas, } 3 x / \text { semana; } 12 \text { exercícios } \\
\text { (MMSS, MMII e Tronco); } 3 \text { séries de } 8 \\
\text { a } 12 \text { RM para MMSS e MMII e } 3 \text { séries } \\
\text { de } 50 \text { reps para o tronco com o peso } \\
\text { corporal }\end{array}$ & Flexímetro & Não informado & $\begin{array}{l}\uparrow \leftrightarrow \text { MMSS } \\
\stackrel{\leftrightarrow}{\text { MMII }} \\
\uparrow \text { Tronco }\end{array}$ \\
\hline $\begin{array}{l}\text { Santos } \\
\text { et al. }{ }^{4}\end{array}$ & $\begin{array}{l}\text { Controle } \\
\text { TF (alternado/ } \\
\text { segmento) } \\
\text { TF (agonista/ } \\
\text { antagonista) }\end{array}$ & $\begin{array}{l}\mathrm{n}=24(\mathrm{M}) \\
\text { INAT } \\
25 \text { anos }\end{array}$ & $\begin{array}{l}8 \text { semanas, } 3 \times \text { /semana; } 9 \text { exercícios } \\
\text { (MMII, MMSS e Tronco); } 3 \text { séries de } 10 \\
\text { a } 12 \text { RM para MMII e MMSS e } 3 \text { séries } \\
\text { de } 15 \text { a } 20 \text { reps para exercícios com o } \\
\text { tronco }\end{array}$ & Goniômetro & $\uparrow$ & $\begin{array}{l}\uparrow \text { MMSS } \\
\uparrow \text { Tronco }\end{array}$ \\
\hline $\begin{array}{l}\text { Potier } \\
\text { et al. }^{3}\end{array}$ & $\begin{array}{l}\text { Controle } \\
\text { TF }\end{array}$ & $\begin{array}{l}n=22(H / M) \\
28 \text { anos }\end{array}$ & $\begin{array}{l}8 \text { semanas, } 3 x / \text { semana; flexão de } \\
\text { pernas (MMII); } 3 \text { séries de 1-8 reps com } \\
\text { a carga inicial de } 1 \text { RM }\end{array}$ & Goniômetro & $\uparrow$ & $\uparrow M M I I$ \\
\hline $\begin{array}{l}\text { Monteiro } \\
\text { et al. }{ }^{23}\end{array}$ & $\begin{array}{l}\text { Controle } \\
\text { TF (circuito) }\end{array}$ & $\begin{array}{l}\mathrm{n}=20(\mathrm{M}) \\
37 \text { anos }\end{array}$ & $\begin{array}{l}10 \text { semanas, } 3 x / \text { semana; } 7 \text { exercícios } \\
\text { (MMII, MMSS e Tronco); } 3 \text { séries de } 8 \text { a } \\
12 \text { RM para MMII e MMSS e de } 15 \text { a } 20 \\
\text { RM para o tronco }\end{array}$ & Flexímetro & $\uparrow$ & $\begin{array}{l}\leftrightarrow \text { MMSS } \\
\uparrow \text { MMII } \\
\uparrow \text { Tronco }\end{array}$ \\
\hline $\begin{array}{l}\text { Zavanela } \\
\text { et al. }{ }^{9}\end{array}$ & $\begin{array}{l}\text { Controle } \\
\text { TF }\end{array}$ & $\begin{array}{l}\mathrm{n}=132(\mathrm{H}) \\
\text { Meia idade }\end{array}$ & $\begin{array}{l}24 \text { semanas, } 3-4 x / \text { semana; } 12 \\
\text { exercícios (MMII, MMSS e Tronco); } 3 \\
\text { séries de } 12 \text { reps de } 10 \text { a } 12 \text { RM }\end{array}$ & $\begin{array}{l}\text { Teste de } \\
\text { sentar e } \\
\text { alcançar }\end{array}$ & $\uparrow$ & $\uparrow \mathrm{MMII}$ e tronco \\
\hline $\begin{array}{l}\text { Adams } \\
\text { et al. }{ }^{16}\end{array}$ & $\begin{array}{l}\text { Controle } \\
\text { TF }\end{array}$ & $\begin{array}{l}\mathrm{n}=19(\mathrm{M}) \\
51 \text { anos }\end{array}$ & $\begin{array}{l}8 \text { semanas, } 2 x / \text { semana; } 9 \text { exercícios } \\
\text { (MMSS e MMII); } 1 \text { a } 3 \text { séries de } 8 \text { a } 10 \\
\text { reps entre } 70 \text { e } 80 \% \text { de } 1 \text { RM }\end{array}$ & $\begin{array}{l}\text { Teste de } \\
\text { sentar e } \\
\text { alcançar }\end{array}$ & $\uparrow$ & $\leftrightarrow \mathrm{MMII}$ \\
\hline
\end{tabular}

TF - Treinamento de força. MMII - membros inferiores; MMSS - membros superiores; RM - repetição máxima; BI - baixa intensidade; MI - moderada intensidade; Al - alta intensidade; reps - repetições. 
TABELA 2 - Características da amostra, procedimentos metodológicos e principais resultados dos estudos sobre o efeito do treinamento de força na flexibilidade de idosos.

\begin{tabular}{|c|c|c|c|c|c|c|}
\hline Autor(es) & Grupos & Amostra & Protocolo de treinamento & $\begin{array}{l}\text { Método/ } \\
\text { Instrumento }\end{array}$ & $\begin{array}{l}\text { Comportamento } \\
\text { da força muscular }\end{array}$ & $\begin{array}{l}\text { Efeito após o } \\
\text { treinamento }\end{array}$ \\
\hline $\begin{array}{l}\text { Vale } \\
\text { et al. }{ }^{15}\end{array}$ & $\begin{array}{l}\text { Controle } \\
\text { TF }\end{array}$ & $\begin{array}{l}n=22(M) \\
65 \text { anos }\end{array}$ & $\begin{array}{l}4 \text { semanas, } 2 x / \text { semana; } 7 \text { exercícios (MMSS e } \\
\text { MMII); } 2 \text { séries de } 15 \text { reps } 50 \% \text { de } 1 \text { RM }+12 \\
\text { semanas, } 2 x / \text { semana; } 7 \text { exercícios (MMSS e } \\
\text { MMII); } 2 \text { séries de } 8 \text { a } 10 \text { reps entre } 75 \text { e } 85 \% \text { de } \\
1 \text { RM }\end{array}$ & Goniômetro & $\uparrow$ & $\begin{array}{l}\uparrow M M S S \\
\uparrow M M I I\end{array}$ \\
\hline $\begin{array}{l}\text { Gonçalves } \\
\text { et al. }{ }^{30}\end{array}$ & $\begin{array}{l}\text { Controle } \\
\text { TF }\end{array}$ & $\begin{array}{l}n=19(\mathrm{H} / \mathrm{M}) \\
65 \text { anos }\end{array}$ & $\begin{array}{l}8 \text { semanas, } 3 x / \text { semana; } 7 \text { exercícios (MMSS, MMII } \\
\text { e tronco); } 3 \text { séries de } 10 \text { a } 12 \text { RM para MMSS e } \\
\text { MMII e } 2 \text { séries de } 15 \text { reps para o abdome com o } \\
\text { peso corporal }\end{array}$ & Flexímetro & Não informado & $\begin{array}{l}\leftrightarrow \text { MMSS } \\
\uparrow \text { MMII } \\
\uparrow \text { Tronco }\end{array}$ \\
\hline $\begin{array}{l}\text { Barbosa } \\
\text { et al. }{ }^{28}\end{array}$ & $\begin{array}{l}\text { Controle } \\
\text { TF }\end{array}$ & $\begin{array}{l}n=19(M) \\
66 \text { anos }\end{array}$ & $\begin{array}{l}10 \text { semanas, } 3 x / \text { semana; } 8 \text { exercícios (MMSS, } \\
\text { MMII e Tronco); } 3 \text { a } 5 \text { séries de } 6 \text { a } 15 \text { reps com } \\
\text { intensidade entre } 6 \text { - } 7 \text { RM para MMSS e MMII e } \\
\text { entre } 10 \text { e } 11 \text { RM para o tronco }\end{array}$ & $\begin{array}{l}\text { Teste de sentar } \\
\text { e alcançar }\end{array}$ & Não informado & $\begin{array}{l}\uparrow \text { MMII e } \\
\text { tronco }\end{array}$ \\
\hline $\begin{array}{l}\text { Fatouros } \\
\text { et al. }{ }^{24}\end{array}$ & $\begin{array}{l}\text { Controle } \\
\text { TF }\end{array}$ & $\begin{array}{l}n=32(H) \\
70 \text { anos }\end{array}$ & $\begin{array}{l}16 \text { semanas, } 3 \times \text { /semana; } 10 \text { exercícios (MMSS, } \\
\text { MMII e Tronco); } 2 \text { a } 4 \text { séries de } 6 \text { a } 10 \text { reps entre } \\
55 \text { e } 80 \% \text { de } 1 \text { RM }\end{array}$ & $\begin{array}{l}\text { Goniômetro } \\
\text { Teste de sentar } \\
\text { e alcançar }\end{array}$ & $\uparrow$ & $\begin{array}{l}\uparrow \text { MMSS } \\
\uparrow \text { Global }\end{array}$ \\
\hline $\begin{array}{l}\text { Fatouros } \\
\text { et al. }{ }^{14}\end{array}$ & $\begin{array}{l}\text { Controle } \\
\text { TF (BI) } \\
\text { TF (MI) } \\
\text { TF (AI) }\end{array}$ & $\begin{array}{l}\mathrm{n}=48(\mathrm{H}) \\
70 \text { anos }\end{array}$ & $\begin{array}{l}\text { Abdome e tronco: } 4 \text { semanas, } 1 \text { série de } 6 \text { reps }+8 \\
\text { semanas, } 2 \text { séries de } 8 \text { reps }+8 \text { semanas, } 3 \text { séries } \\
\text { de } 10 \text { reps }+4 \text { semanas, } 4 \text { séries de } 10-12 \text { reps } \\
\text { peso do tronco } \\
8 \text { exercícios (MMSS, MMSII): } 8 \text { semanas, } 2 \text { séries } \\
\text { de } 15 \text { reps (BI), } 10 \text { reps (MD), } 8 \text { reps (AI) a } 45-50 \% \\
1 \text { RM (BI), } 60-65 \% 1 \text { RM (MI), } 80-85 \% 1 \text { RM (AI) + } \\
16 \text { semanas, } 3 \text { séries com as mesmas cargas e } \\
\text { repetições das semanas anteriores }\end{array}$ & $\begin{array}{l}\text { Goniômetro } \\
\text { Teste de sentar } \\
\text { e alcançar } \\
\text { modificado }\end{array}$ & $\uparrow$ & $\begin{array}{l}\uparrow \text { MMSS } \\
\uparrow \text { MMII } \\
\uparrow \text { Tronco }\end{array}$ \\
\hline
\end{tabular}

TF - Treinamento de força. MMII - membros inferiores; MMSS - membros superiores; RM - repetição máxima; BI - baixa intensidade; $\mathrm{MI}$ - moderada intensidade; Al - alta intensidade; reps - repetições.

\section{DISCUSSÃO}

Os resultados desta revisão sistemática indicam que a faixa etária pode ser um fator interveniente para a resposta do treinamento de força na flexibilidade, visto que os estudos foram unânimes em reportar aumentos de flexibilidade em idosos enquanto que, em adultos jovens, os resultados foram controversos. Além disso, por meio deste estudo foi possível identificar que os estudos que observaram aumentos da flexibilidade em adultos jovens utilizaram instrumentos de avaliação mais precisos.

Dos onze estudos que analisaram os efeitos do treinamento de força sobre a flexibilidade de adultos jovens, quatro verificaram manutenção nos níveis de flexibilidade e sete observaram aumentos dessa variável em pelo menos uma articulação. Interessantemente, essa controvérsia parece não estar relacionada com o período de intervenção, a frequência de treinamento semanal e o volume de treino empregado nos estudos, pois estes foram semelhantes entre os estudos que observaram e os que não observaram efeito significante. Dessa forma, não foi possível identificar protocolos que poderiam influenciar nas respostas da flexibilidade com o treinamento de força.

Um dos aspectos que deve ser considerado na interpretação dos resultados da presente revisão é o método/instrumento utilizado para avaliação da flexibilidade. De fato, todos os estudos que utilizaram o goniômetro ou o flexímetro verificaram aumentos nos níveis de flexibilidade com o treinamento de força em pelo menos uma articulação. Por outro lado, os estudos que utili- 
zaram o teste de sentar e alcançar apresentaram resultados controversos. Sabe-se que o teste de sentar e alcançar pode ser influenciado por características antropométricas, o que não ocorre em testes que avaliam ângulo e amplitude de movimento a partir de pontos anatômicos específicos ${ }^{25}$. Além disso, o teste de sentar e alcançar quantifica a flexibilidade de diferentes articulações simultaneamente em um único movimento, enquanto o flexímetro e o goniômetro analisam cada articulação de maneira isolada. Dessa forma, é possível que os efeitos do treinamento de força sejam evidenciados apenas em articulações específicas, o que precisa ser investigado mais profundamente. Seria interessante que estudos futuros analisassem os efeitos do treinamento de força utilizando diferentes métodos para avaliação da flexibilidade.

Todos os estudos com idosos incluídos nessa revisão verificaram aumentos na flexibilidade após o treinamento de força, o que pode ser atribuído, principalmente, à reduzida mobilidade articular observada nesse grupo etário. Sabe-se que o grau de mobilidade está diretamente relacionado com a autonomia funcional de idosos. Assim, o aumento da flexibilidade com o treinamento de força pode minimizar os efeitos deletérios do envelhecimento, repercutindo numa melhor execução das atividades da vida diária ${ }^{7,26}$. Além disso, o aumento na amplitude dos movimentos para a execução dos exercícios de força reduz os riscos de lesões músculoarticulares e a rigidez dos músculos desses indivíduos?

Embora os mecanismos envolvidos no aumento da flexibilidade com o treinamento de força não estejam totalmente esclarecidos, o aumento da força parece influenciar nessa resposta. Como todos os testes para avaliação da flexibilidade são ativos, de forma que o indivíduo precisa utilizar a força para conseguir uma melhor amplitude de movimento, aumentos na força poderiam repercutir em aumentos de flexibilidade. De fato, nove estudos, sendo três com idosos $^{14,15,24}$ e seis com adultos jovens ${ }^{1-4,9,23}$, avaliaram a força muscular dos participantes e identificaram um aumento da força atrelado a uma melhora dos níveis de flexibilidade. Fisiologicamente, os mecanismos que têm sido propostos envolvem a redução da taxa de disparo do fuso muscular durante a avaliação da flexibilidade ${ }^{27}$ após a realização de algumas sessões de treinamento de força. Por conta disso, há um aumento na amplitude do movimento e diminuição do incômodo sofrido durante a realização do teste máximo de flexibilidade.

Com relação aos métodos empregados pelos estudos, é importante destacar que, dos 16 estudos incluídos na revisão, dois ${ }^{28,29}$ reportaram que a alocação dos grupos de intervenção não foi aleatória e sete não forneceram informações sobre esta alocação ${ }^{1,2,4,14,18,24,30}$. Além disso, a minoria dos estudos forneceu informações que asseguram a maior qualidade das medidas realizadas, como a familiarização dos voluntários aos testes realizados ${ }^{16,23}$, erro técnico ${ }^{22}$ e reprodutibilidade das medidas ${ }^{4,9}$, o que pode influenciar os resultados e a validade interna do estudo. Esses fatores devem ser considerados em estudos futuros visando assegurar a qualidade das medidas realizadas.

Esta revisão apresenta algumas limitações. Foram incluídos artigos disponíveis em cinco bases de dados que investigaram o efeito do treinamento de força na flexibilidade. É possível que outros estudos sobre a temática tenham sido indexados em outras bases de dados e não foram incluídos nesta revisão. Além disso, não foram realizadas buscas em bibliotecas digitais ou bancos de teses e dissertações. 
Conclui-se que a faixa etária é um fator interveniente para o efeito do treinamento de força na flexibilidade. Em idosos, o treinamento de força aumentou a flexibilidade, independente do protocolo utilizado e do instrumento/ método de avaliação. Em adultos jovens, resultados controversos foram observados, entretanto, efeitos significativos parecem ser identificados quando a avaliação é feita em articulações específicas.

\section{Agradecimentos}

Os autores agradecem ao Conselho Nacional de Desenvolvimento Científico e Tecnológico (CNPq) pela bolsa de produtividade em pesquisa (R.M.R.D.) e à Coordenação de Aperfeiçoamento de Pessoal de Nível Superior (CAPES) pelas bolsas de mestrado (A.L.M., M.A.C. e A.H.R.A.) outorgadas.

\section{Contribuição dos autores}

M.A.C., A.L.M. e R.M.R. delinearam o estudo e lideraram a redação do manuscrito. M.A.C. e A.L.M. realizaram as pesquisas bibliográficas. M.A.C., A.L.M. e B.R.C. fizeram a extração dos dados. B.R.C., A.H.R.A. e R.M.R. auxiliaram na interpretação dos resultados encontrados. M.A.C., A.L.M., B.R.C., A.H.R.A. e R.M.R. participaram da redação do manuscrito e aprovaram a versão final.

\section{REFERÊNCIAS}

1. Mahieu NN, McNair P, Cools A, Effect of eccentric training on the plantar flexor muscle-tendon tissue properties. Med and Sci in Sports and Exerc 2008; 40: 117-123.

2. Morton SK, Whitehead JR, Brinkert RH, and Caine DJ, Resistance training vs. static stretching: effects on flexibility and strength. J Strength Cond Res 2011; 25: 3391-3398.

3. Potier TG, Alexander CM, and Seynnes OR, Effects of eccentric strength training on biceps femoris muscle architecture and knee joint range of movement. Eur J Appl Physiol 2009; 105: 939-944.

4. Santos E, Rhea MR, Simao R, Influence of moderately intense strength training on flexibility in sedentary young women. J Strength Cond Res 2010; 24: 3144-3149.

5. American College of Sports Medicine, American College of Sports Medicine Position Stand. Exercise and physical activity for older adults. Med Sci Sports Exerc 1998; 30: 992-1008.

6. Dias RMR, Gurjão ALD, and Marucci MFN, Benefícios do treinamento com pesos para aptidão física de idosos. Acta Fisiátr 2006; 13: 90-95.

7. American College of Sports Medicine, American College of Sports Medicine Position Stand. The recommended quantity and quality of exercise for developing and maintaining cardiorespiratory and muscular fitness, and flexibility in healthy adults. Med Sci Sports Exerc 1998; 30: 975-991.

8. Santos CFd, Crestan TA, Picheth DM, Efeito de 10 semanas de treinamento com pesos sobre indicadores da composição corporal. R bras Ci e Mov 2002; 10: 79-84.

9. Zavanela PM, Crewther BT, Lodo L, Health and fitness benefits of a resistance training intervention performed in the workplace. J Strength Cond Res 2012; 26: 811-817.

10. Badley EM, Wagstaff S, and Wood PH, Measures of functional ability (disability) in arthritis in relation to impairment of range of joint movement. Ann Rheum Dis 1984; 43: 563-569.

11. Barbosa AR, Souza JM, Lebrao ML, Functional limitations of Brazilian elderly by age and gender differences: data from SABE Survey. Cad Saúde Pública 2005; 21: 1177-1185.

12. Brown DA and Miller WC, Normative data for strength and flexibility of women throughout life. Eur J Appl Physiol Occup Physiol 1998; 78: 77-82.

13. Matsudo SM, Matsudo VKR, Neto TLB, and Araújo TL, Evolução do perfil neuromotor e capacidade funcional de mulheres fisicamente ativas de acordo com a idade cronológica. Rev Bras Med Esporte 2003; 9: 365-376. 
14. Fatouros IG, Kambas A, Katrabasas I, Resistance training and detraining effects on flexibility performance in the elderly are intensity-dependent. J Strength Cond Res 2006; 20: 634-642.

15. Vale RGS, Barreto ACG, Novaes JS, and Dantas EHM, Efeitos do treinamento resistido na força máxima, na flexibilidade e na autonomia funcional de mulheres idosas. Rev bras cineantropom desempenho hum 2006; 8: 52-58.

16. Adams KJ, Swank AM, Berning JM, Progressive strength training in sedentary, older African American women. Med Sci Sports Exerc 2001; 33: 1567-1576.

17. Aquino CF, Fonseca ST, Goncalves GG, Stretching versus strength training in lengthened position in subjects with tight hamstring muscles: a randomized controlled trial. Man Ther 2010; 15: 26-31.

18. Kim E, Dear A, Ferguson SL, Effects of 4 weeks of traditional resistance training vs. superslow strength training on early phase adaptations in strength, flexibility, and aerobic capacity in college-aged women. J Strength Cond Res 2011; 25: 3006-3013.

19. Kubo K, Kanehisa H, Miyatani M, Effect of low-load resistance training on the tendon properties in middle-aged and elderly women. Acta Physiol Scand 2003; 178: 25-32.

20. Narici MV, Maffulli N, and Maganaris CN, Ageing of human muscles and tendons. Disabil Rehabil 2008; 30: 1548-1554.

21. Araújo VLd, Carvalhais VOdC, Ocarino JdM, Efeito dos exercícios de fortalecimento e alongamento sobre a rigidez tecidual passiva. Fisioterapia em Movimento 2012; 25: 869-882.

22. Cyrino ES, Oliveira AR, Leite JC, Comportamento da flexibilidade após 10 semanas de treinamento com pesos. Rev Bras Med Esporte 2004; 10: 233-237.

23. Monteiro WD, Simao R, Polito MD, Influence of strength training on adult women's flexibility. J Strength Cond Res 2008; 22: 672-677.

24. Fatouros IG, Taxildaris K, Tokmakidis SP, The effects of strength training, cardiovascular training and their combination on flexibility of inactive older adults. Int J Sports Med 2002; 23: 112-119.

25. Cornbleet SL and Woolsey NB, Assessment of hamstring muscle length in school-aged children using the sit-and-reach test and the inclinometer measure of hip joint angle. Phys Ther 1996; 76: 850-855.

26. Barak Y, Ayalon M, and Dvir Z, Transferability of strength gains from limited to full range of motion. Med Sci Sports Exerc 2004; 36: 1413-1420.

27. De Luca CJ and Erim z, Common drive of motor units in regulation of muscle force. Trends Neurosci 1994; 17: 299-304.

28. Barbosa AR, Santarém JM, Filho WJ, and Marucci MF, Effects of resistance training on the sit-and-reach test in elderly women. J Strength Cond Res 2002; 16: 14-18.

29. Nobrega AC, Paula KC, and Carvalho AC, Interaction between resistance training and flexibility training in healthy young adults. J Strength Cond Res 2005; 19: 842-846.

30. Gonçalves R, Gurjão ALD, and Gobbi S, Efeitos de oito semanas do treinamento de força na flexibilidade de idosos. Rev bras cineantropom desempenho hum 2007; 9: 145-153.

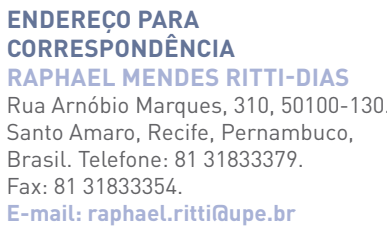

